\title{
BMJ Open Preoxygenation with non-invasive ventilation versus high-flow nasal cannula oxygen therapy for intubation of patients with acute hypoxaemic respiratory failure in ICU: the prospective randomised controlled FLORALI-2 study protocol
}

\author{
Jean-Pierre Frat, ${ }^{1,2,3}$ Jean-Damien Ricard, ${ }^{4,5,6}$ Rémi Coudroy, ${ }^{1,2,3}$ René Robert, ${ }^{1,2,3}$ \\ Stéphanie Ragot, ${ }^{2,7,8}$ Arnaud W Thille, ${ }^{1,2,3}$ on-behalf-of REVA network
}

To cite: Frat J-P,

Ricard J-D, Coudroy R, et al. Preoxygenation with noninvasive ventilation versus high-flow nasal cannula oxygen therapy for intubation of patients with acute hypoxaemic respiratory failure in ICU: the prospective randomised controlled FLORALI-2 study protocol. BMJ Open 2017;7:e018611. doi:10.1136/ bmjopen-2017-018611

\section{- Prepublication history for} this paper is available online. To view these files, please visit the journal online (http://dx.doi. org/10.1136/bmjopen-2017018611).

Received 24 July 2017 Revised 6 November 2017 Accepted 8 November 2017

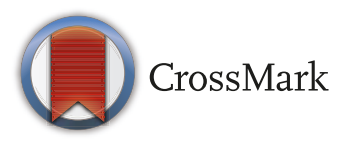

For numbered affiliations see end of article.

Correspondence to Dr Jean-Pierre Frat; jean-pierre.frat@chu-poitiers.fr

\section{ABSTRACT}

Introduction Endotracheal intubation in intensive care unit (ICU) is a procedure at high risk of lifethreatening complications. Among them, severe oxygen desaturation, usually defined as a drop of pulse oxymetry $\left(\mathrm{SpO}_{2}\right)$ below $80 \%$, is the most common. Preoxygenation enables delaying oxygen desaturation occurring during apnea induced by anaesthetic drugs. Data suggest that non-invasive ventilation (NIV) or high-flow nasal cannula (HFNC) oxygen therapy could further increase $\mathrm{PaO}_{2}$ before intubation procedure and prevent oxygen desaturation episodes as compared with standard oxygen. However, no recommendation favours one technique rather than the other, since they have never been compared. Hence, whether a strategy of preoxygenation with NIV or HFNC is more effective than the other in patients with acute hypoxaemic respiratory failure remains to be established.

Methods and analysis The FLORALI-2 study is a multicentre randomised controlled trial comparing a preoxygenation strategy with either NIV or HFNC in patients with acute hypoxaemic respiratory failure needing intubation in ICU. The 320 patients will be randomised with a ratio 1:1 in two groups according to the strategy of preoxygenation. The primary outcome is the occurrence of an episode of severe oxygen desaturation defined by a drop of $\mathrm{SpO}_{2}$, below $80 \%$ during the intubation procedure. Secondary outcomes include feasibility of the two strategies, immediate and late complications related to intubation.

Ethics and dissemination The study has been approved by the central ethics committee (Ethics Committee Ouest-III, Poitiers, France) and patients will be included after informed consent. The results will be submitted for publication in peer-reviewed journals.

Trial registration number NCT02668458; Pre-results.
Strengths and limitations of the study

- Largest prospective multicentre randomised controlled study comparing two preoxygenation strategies in this field.

- Assessment of primary outcome, severe hypoxaemia during the intubation procedure: in order to improve measurement reliability in all centres, a dedicated portable $\mathrm{SpO}_{2}$ monitor with single-patient-use digital sensors is used for each eligible patient to ensure that all centres have equivalent monitoring; secondarily, the downloaded data will be reviewed by a committee unaware of the study groups so as to analyse the events occurring during intubation procedure.

- Blinding: the individual study assignments of the patients will not be masked, however, given the characteristics of the two strategies under evaluation, a double-blind trial is not possible.

\section{INTRODUCTION}

\section{Background and rationale}

Endotracheal intubation of critically ill patients in the intensive care unit (ICU) is a procedure at high risk of life-threatening complications including severe hypoxaemia, cardiac arrhythmias and cardiovascular collapse that may-in some instances-unfortunately lead to cardiac arrest. Severe hypoxaemia, that is, blood oxygen desaturation, usually defined as a drop of pulse oxymetry $\left(\mathrm{SpO}_{2}\right)$ below $80 \%$, is a common complication occurring in $20 \%-25 \%$ of the cases during the procedure of intubation in ICU, ${ }^{12}$ preferentially in patients with hypoxaemia intubated for acute respiratory failure..$^{1-5}$ 
Preoxygenation should be performed systematically before intubation with the aim of increasing arterial oxygen tension $\left(\mathrm{PaO}_{2}\right)$ and of delaying the oxygen desaturation occurring during apnea induced by anaesthetic drugs. Adequate preoxygenation enables increased oxygen body stores, mainly in the pulmonary functional residual capacity, and subsequent $\mathrm{PaO}_{2}$ by breathing high $\mathrm{FiO}_{2}$ during deep breaths. ${ }^{6}$ Breathing through a valve-bag facemask during at least $3-5 \mathrm{~min}$ is the usual method to carry out preoxygenation in the operating room. However, other techniques using non-invasive ventilation (NIV) or high-flow nasal cannula (HFNC) oxygen therapy could be used, especially for patients with hypoxaemia in ICU, to further increase $\mathrm{PaO}_{2}$ before intubation procedure and to prevent oxygen desaturation. ${ }^{7}$ Indeed, HFNC enables the delivery of continuous high gas flow via nasal prongs resulting in higher $\mathrm{FiO}_{2}$ than with standard oxygen. ${ }^{8}$ Another theoretical advantage of HFNC may consist in maintaining oxygenation during the apneic phase of intubation, and thereby avoiding oxygen desaturation. ${ }^{7}$ Although in a prospective before-after study the use of HFNC with a flow of $60 \mathrm{~L} /$ min resulted in lower incidence of severe oxygen desaturation during intubation than with standard oxygen, ${ }^{9}$ these encouraging results have not been confirmed in the different randomised controlled trials (RCT) carried out to date. ${ }^{10-12}$

Preoxygenation with NIV is another method of preoxygenation that enables delivery of inspired $\mathrm{FiO}_{2}$ reaching up to $100 \%$ in the absence of leaks, and it may significantly increase end-expiratory lung volume due to the alveolar recruitment induced by positive airway pressure. ${ }^{13}$ Given these beneficial effects on oxygenation, NIV may be an effective method for preoxygenation preventing oxygen desaturation during the procedure of intubation. However, to date, only one RCT including a small sample of patients has been conducted in ICU. ${ }^{14}$ This study found a decrease in episodes of severe oxygen desaturation with NIV as compared with standard valve-bag mask for the preoxygenation of patients with acute hypoxaemic respiratory failure. ${ }^{14}$ In a more recent retrospective study, no difference was found between preoxygenation with NIV and HFNC. ${ }^{15}$ However, the small sample size of these two studies do not allow for conclusions. Finally, one recent study showed that combining NIV and HFNC was superior to NIV alone as a means of preventing desaturation, but provided no answer as to whether or not NIV alone was superior to HFNC alone. ${ }^{16}$

\section{Objectives}

We aim to conduct a prospective multicentre randomised controlled trial comparing NIV versus HFNC in a population of patients with acute hypoxaemic respiratory failure needing intubation in ICU, with the hypothesis that preoxygenation with NIV could better prevent severe oxygen desaturation $\left(\mathrm{SpO}_{2}<80 \%\right)^{1}$ during the procedure of intubation as compared with HFNC.

\section{Primary objective}

To compare the number of patients with acute hypoxaemic respiratory failure having an episode of severe oxygen desaturation during the intubation procedure in the ICU, between strategies of preoxygenation performed with NIV versus HFNC.

\section{Secondary objectives}

To compare the highest $\mathrm{SpO}_{2}$ at the end of preoxygenation, the lowest $\mathrm{SpO}_{2}$ during and immediately after the procedure of intubation between the two strategies of preoxygenation.

To compare the number of immediate and late complications between the two methods, and to assess their feasibility.

\section{Trial design}

The FLORALI-2 study is an investigator-initiated, prospective, multicentre, randomised, control, open trial on medical devices comparing a strategy of preoxygenation with either NIV or HFNC for intubation of patients with acute hypoxaemic respiratory failure in ICU. Patients are randomly assigned to the NIV group or HFNC group, with a $1: 1$ ratio.

\section{METHODS: PARTICIPANTS, INTERVENTIONS AND OUTCOMES Study setting}

The FLORALI-2 study is taking place in 28 mixed medical and surgical ICU in France (list on https://clinicaltrials.gov/ct2/show/NCT02668458?term=Florali+2\& rank=1) belonging to the European REVA network and CRICS-TRIGGERSEP network.

\section{Eligibility criteria}

Inclusion criteria

Adult patients admitted to ICU with acute hypoxaemic respiratory failure and requiring endotracheal intubation according to the decision by the medical doctor in charge may be included in the study. Acute hypoxaemic respiratory failure is defined as a respiratory rate above 25 breaths per minute or signs of respiratory distress, and a $\mathrm{PaO}_{2} / \mathrm{FiO}_{2}$ ratio below $300 \mathrm{~mm} \mathrm{Hg}$ regardless the strategy of oxygenation administered (figure 1). The $\mathrm{PaO}_{2} / \mathrm{FiO}_{2}$ ratio will be measured under NIV or HFNC, and calculated under standard oxygen as follows: $\mathrm{FiO}_{2}=0.21+\mathrm{ox}-$ ygen flow rate $\times 0.03 .{ }^{17}$

\section{Exclusion criteria}

Patients fulfilling one or more of the following criteria will not be included: intubation for cardiac arrest, altered consciousness defined by a Glasgow Coma Score $<8$ points, contraindications to NIV, $\mathrm{SpO}_{2}$ not available, age $<18$ years, pregnant or breast-feeding women (figure 1).

\section{Intervention}

Preoxygenation

Patients eligible for inclusion will be randomly assigned to the NIV group or HFNC group (figure 2). 


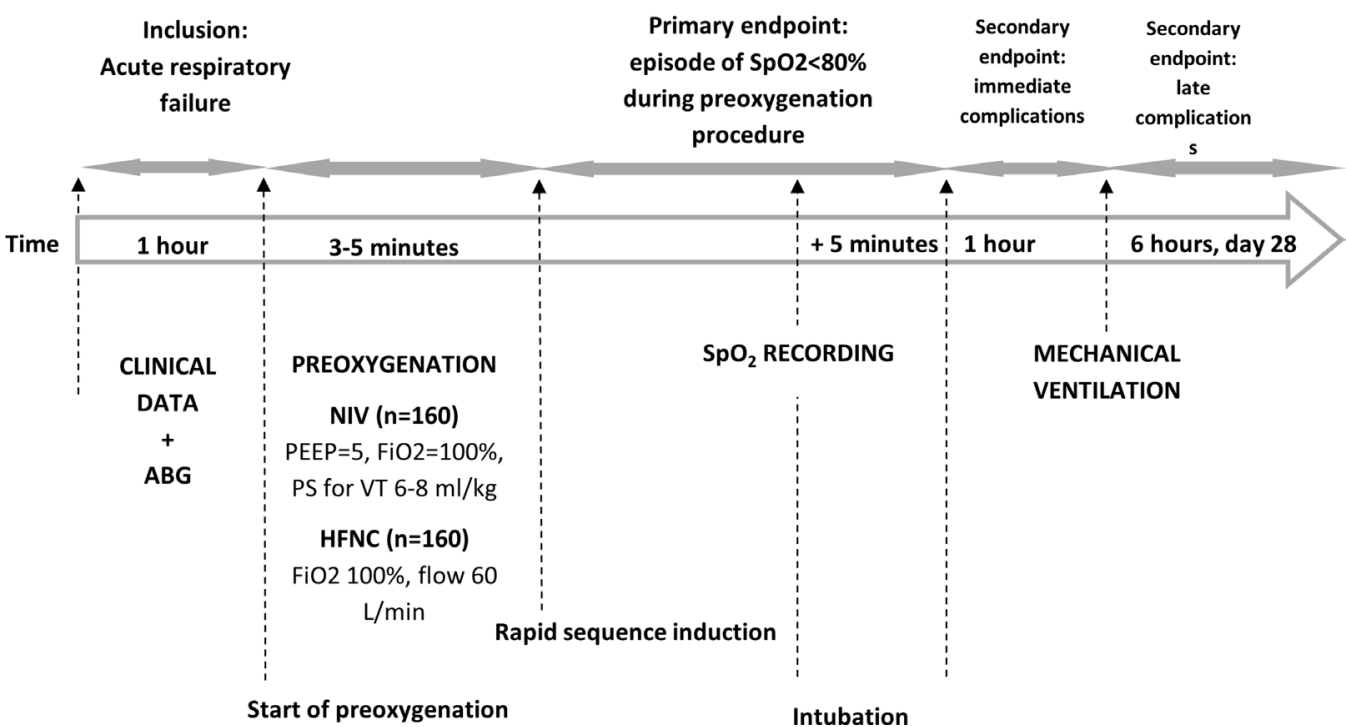

Figure 1 Consort diagram of the FLORALI-2 study. ABG, arterial blood gas; $\mathrm{FiO}_{2}$, fractional inspired oxygen; HFNC, high-flow nasal cannulae oxygen therapy; NIV, non-invasive ventilation; $\mathrm{PaO}_{2} / \mathrm{FiO}_{2}$, partial pressure of arterial oxygen to fractional inspired oxygen ratio; PEEP, positive end-expiratory pressure; PS, pressure support; $\mathrm{SpO}_{2}$, pulse oxymetry.

Preoxygenation will be carried out in a semirecumbent position for $3-5$ min with the device assigned by randomisation (NIV or HFNC), whatever the previous device used for oxygenation.

The interventional arm will consist in applying NIV delivered to the patient with a full-face mask connected to an ICU ventilator. Pressure-support ventilation will be adapted to obtain an expired tidal volume between 6 and $8 \mathrm{~mL} / \mathrm{kg}$ of predicted body weight with a positive end-expiratory pressure (PEEP) level of $5 \mathrm{~cm} \mathrm{H}_{2} \mathrm{O}$ and an $\mathrm{FiO}_{2}$ of $100 \%$.

The control arm will consist in applying HFNC device (Optiflow, Fisher \& Paykel, Auckland, New Zealand) with a gas flow of $60 \mathrm{~L} / \mathrm{min}$ through a heated humidifier (MR 850, Fisher \& Paykel, Auckland, New Zealand) and an $\mathrm{FiO}_{2}$ of at $100 \%$. A particular attention will be paid to perform a jaw thrust so as to maintain patent upper airway.

If the assigned method is ineffective $\left(\mathrm{SpO}_{2}<80 \%\right)$ or cannot be applied (intolerance, agitation...), the investigator will be free to switch to an alternative method or to intubate immediately. In all cases, the patient will be considered as having failed in the assigned preoxygenation method and will undergo intention-to-treat analysis.

An intervention care bundle management including the following measures is proposed to all the participating centres for the intubation procedure ${ }^{3}$ : the presence of two operators, systematic fluid loading before intubation in absence of cardiogenic pulmonary oedema and rapid-sequence induction using hypnomidate $(0.2-0.3 \mathrm{mg}$ / $\mathrm{kg})$ or ketamine $(1.5-3 \mathrm{mg} / \mathrm{kg})$ combined with rocuronium $(0.6-1 \mathrm{mg} / \mathrm{kg})$ or succinylcholine $(1 \mathrm{mg} / \mathrm{kg})$. In case of unsuccessful intubation, the following algorithm according to a given centre's procedure is proposed: an intubating stylet will be used first, then videolaryngoscopy, an intubation laryngeal mask airway, and finally fiberscopy and rescue percutaneous or surgical tracheostomy. After endotracheal intubation, the patient will be mechanically ventilated with a tidal volume between 6 and $8 \mathrm{~mL} / \mathrm{kg}$, a respiratory rate of $25-30$ breaths/min, a PEEP of $5 \mathrm{~cm} \mathrm{H}_{2} \mathrm{O}$ and an $\mathrm{FiO}_{2}$ of $100 \%$.

\section{Outcomes}

\section{Primary outcome measure}

The primary outcome is the occurrence of an episode of severe oxygen desaturation defined by a drop of $\mathrm{SpO}_{2}$ below $80 \%$, during the intubation procedure carried out by NIV or HFNC.

The intubation procedure lasts from the beginning of rapid-sequence induction (end of preoxygenation) to 5 min after confirmation of the tracheal intubation by capnography and after mechanical ventilation is started.

\section{Secondary outcome measures}

Secondary outcome variables include the following:

- feasibility of the two methods will be evaluated by the operator as satisfaction assessment of the randomly assigned method with a Likert scale model, including the following items: extremely satisfied ( +2 points), very satisfied ( +1 point), somewhat satisfied ( 0 point), slightly satisfied ( -1 point) and not at all satisfied ( -2 points);

- highest $\mathrm{SpO}_{2}$ and proportion of patients reaching an $\mathrm{SpO}_{2}$ at $100 \%$ at the end of preoxygenation;

- inability to obtain an $\mathrm{SpO}_{2}>92 \%$ during preoxygenation;

- lowest $\mathrm{SpO}_{2}$ during intubation;

- immediate complications defined as the following: during difficult intubation ( $>2$ laryngoscopic attempts to place the endotracheal tube into the trachea or as lasting more than $10 \mathrm{~min}$ using conventional laryngoscopy); regurgitation; new infiltrate on chest 


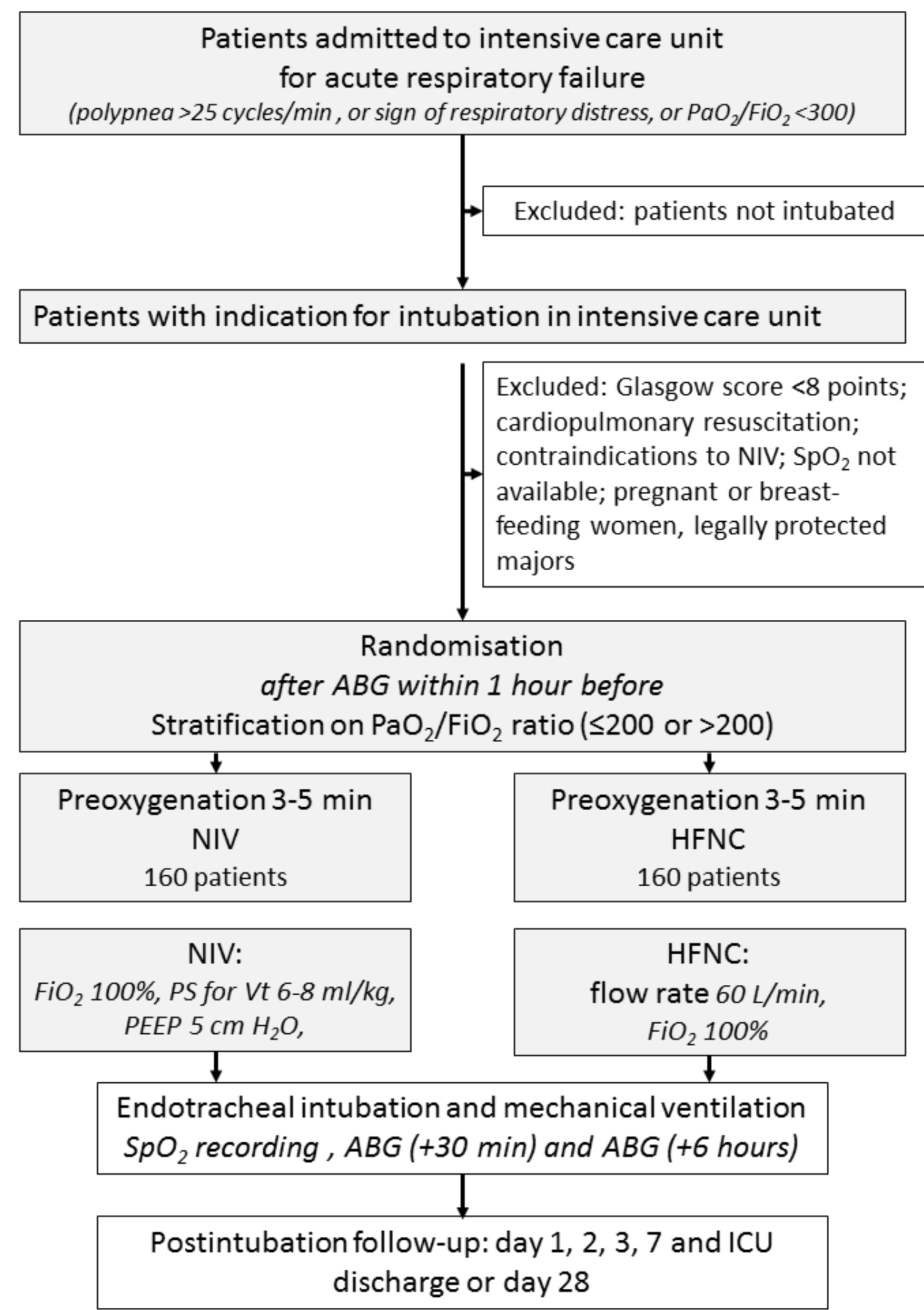

Figure 2 Study design of the FLORALI-2 study. ABG, arterial blood gas; HFNC, high-flow nasal cannulae oxygen therapy; ICU, intensive care unit; NIV, non-invasive ventilation; $\mathrm{PaO}_{2} / \mathrm{FiO}_{2}$, partial pressure of arterial oxygen to fractional inspired oxygen ratio; PEEP, positive end-expiratory pressure; PS, pressure support; $\mathrm{SpO}_{2}$, pulse oxymetry.

radiograph; cardiac arrhythmia, hypotension (systolic blood pressure $<90 \mathrm{~mm} \mathrm{Hg}$, mean blood pressure $<65 \mathrm{~mm} \mathrm{Hg}$ more than $30 \mathrm{~min}$ or requiring volume expansion or vasopressor), cardiac arrest and death;

- late complications defined as the following: radiological infiltrates within the 3 days following intubation, occurrence of ventilator-associated pneumonia; worsening of Sequential Organ Failure Assessment (SOFA) score from day 1 to day 3; duration of mechanical ventilation, ICU length of stay and mortality in ICU and at day 28.

\section{Participant timeline}

Figure 1 shows the Consort diagram of the FLORALI-2 study and table 1 assessments and visits for participants.

\section{Sample size}

The primary outcome is the number of occurrences of an $\mathrm{SpO}_{2}$ below $80 \%$ during the intubation procedure. Three hundred and twenty patients in total, that is, 160 patients per group, will be needed to demonstrate a decrease in absolute difference between the NIV group and HFNC group of $15 \%$. Based on the literature, ${ }^{131214}$ we estimated 


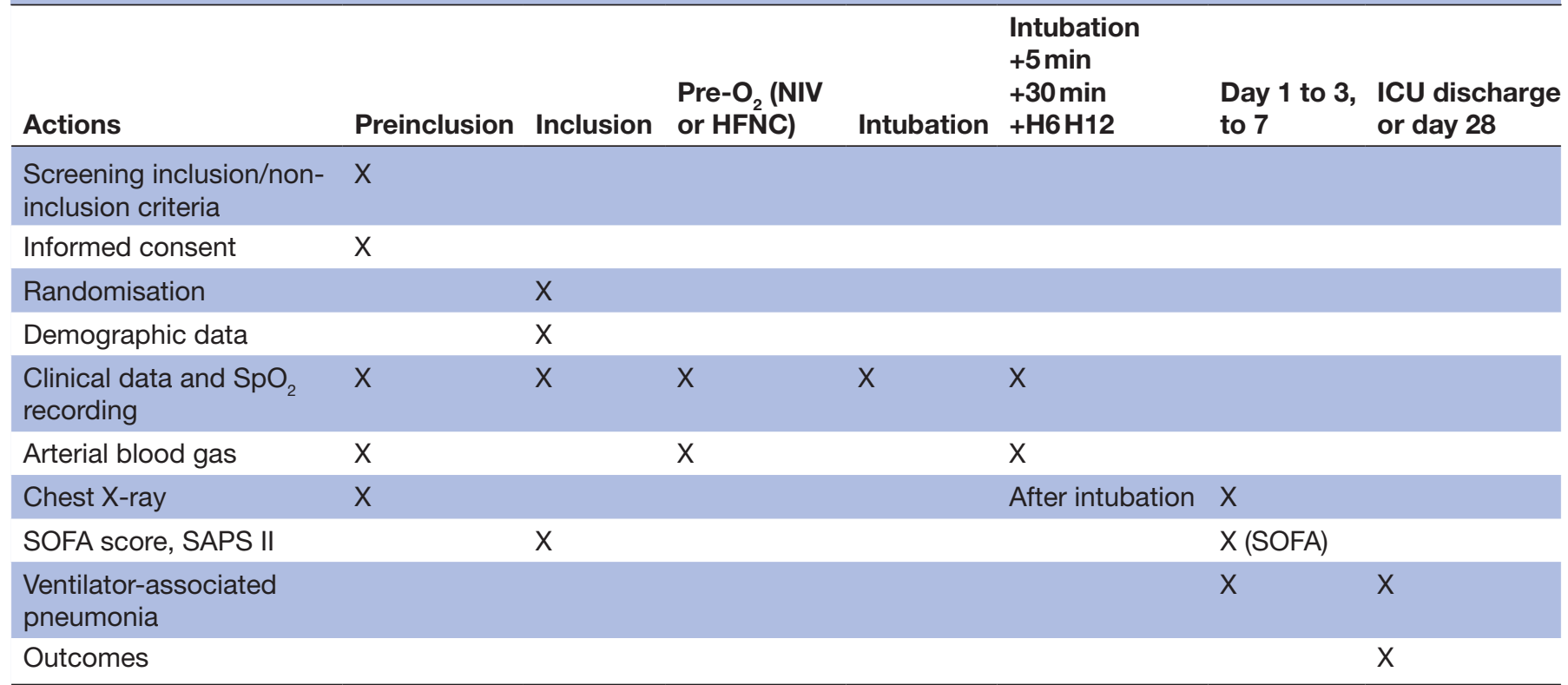

HFNC, high-flow nasal cannulae oxygen therapy; ICU, intensive care unit; NIV, non-invasive ventilation; Pre- $\mathrm{O}_{2}$, preoxygenation; SAPS, Simplified Acute Physiology Score; SOFA, Sequential Organ Failure Assessment; $\mathrm{SpO}_{2}$, pulse oxymetry.

a rate of severe desaturation episodes of $10 \%$ in the NIV group and $25 \%$ in the HFNC group. The $\alpha$ and $\beta$ risks are 0.05 with a bilateral formulation.

\section{Recruitment}

The initial duration of patient enrolment expected will be 2 years, starting in April 2016.

- 2015-2016: approvals from ethics committee and trial tool development (electronic case-report form (e-CRF), randomisation system), participating centres opening and training;

- 2016: inclusion of patients completed in end-December 2016;

- 2017: monitoring of participating centres and queries to investigators; overseen by the steering committee at the REVA Network meetings every 4 months; blind review to determine protocol violation, to define intention-to-treat and per-protocol analysis populations; new queries to investigators, cleaning and closure of the database;

- 2018: data analysis, writing of the manuscript and submission for publication.

\section{METHODS: ASSIGNMENT OF INTERVENTION Allocation and sequence generation}

A computer-generated randomisation will be performed with stratification according to centre and $\mathrm{PaO}_{2} / \mathrm{FiO}_{2}$ ratio $\leq 200$ or $>200$ in a 1:1 ratio, with the use of a centralised web-based management system (Clinfile). Within 1 hour after the validation of inclusion criteria, patients must be randomised and assigned the strategy started (HFNC or NIV).

\section{Blinding}

Although the individual study assignments of the patients will not be masked, the co-ordinating centre and all the investigators will remain unaware of the study group outcomes until the data will be locked.

\section{METHODS: DATA COLLECTION, MANAGEMENT AND ANALYSIS Data collection and management}

Data are collected and recorded on an e-CRF by a trained investigator or research assistant at each centre. A blank copy of the e-CRF can be printed from the e-CRF; this enables the investigator or research assistant to fill it out with the data of the included patients, which will thereby be captured. Once data collection has been completed, the investigator or research assistant shall sign and date the copy. This document will constitute an integral part of the patient's medical records; as such, it shall be retained permanently. Data recorded in the e-CRF that originate in source documents must be consistent with each other; if they are not, the differences have got to be justified and documented.

With the aim of homogenising measurement quality, the recording and collection of patients' data in all centres, a dedicated portable $\mathrm{SpO}_{2}$ monitor (Covidien, Nelcor DS 100A) and single-patient-use digital sensors (Covidien, Max-A-I) will be provided to all the participating centres to ensure that all centres have equivalent monitoring. $\mathrm{SpO}_{2}$ will be recorded from the beginning of preoxygenation to 1 hour after intubation with the same device in all included patients; secondarily, the downloaded data will be reviewed by a committee unaware of the study groups so as to analyse the events occurring during intubation procedure. 
Before intubation, the following data are collected and registered: demographic data, comorbidities, type of admission, diagnostic of respiratory failure, main reason for endotracheal intubation, oxygen support before the decision of intubation, clinical parameters, severity scores using the Simplified Acute Physiology Score (SAPS) II and the SOFA score at inclusion and 24 hours after admission, chest X-ray description (parenchyma involvement of the four quadrants, normal aspect, interstitial infiltrates, alveolar infiltration or condensation) and arterial blood gas.

After randomisation, the following parameters are recorded: nature and number of operators and their training, clinical parameters, $\mathrm{SpO}_{2}$ variations (from preoxygenation to $\mathrm{H} 1$ after intubation, at H6-12), arterial blood gases (30 min and at H6-12 after intubation), criteria of difficult intubation evaluated by the MACOCHA score ${ }^{18}$; during the intubation procedure, lowest $\mathrm{SpO}_{2}$, drop $<80 \%$ of $\mathrm{SpO}_{2}, \mathrm{SpO} 2 \geq 92 \%, \mathrm{SpO} 2$ at $100 \%$, feasibility of preoxygenation strategy, duration of preoxygenation, laryngoscopy, number of attempts, Cormack-Lehane grade, ${ }^{19}$ traction force on laryngoscope, Sellick manoeuvre, modified Intubation Difficulty Scale score. ${ }^{20}$ Immediate complications will be collected, including moderate complications, regurgitation (aspiration of gastric content), oesophageal intubation, cardiac arrhythmia (supra or ventricular arrhythmia) requiring therapy, dangerous agitation (Richmond Agitation-Sedation Scale score $>3$ ), dental injury; severe complications, arterial hypotension and treatments initiated, cardiac arrest, death; new infiltrate, gastric distension on postendotracheal intubation chest X-ray.

During ICU stay or at day 28, the following data will be recorded: SAPS II, SOFA score (within 24 hours after admission), day 1 to day 3 , ventilator-associated pneumonia, ICU length of stay, mechanical ventilation duration, ICU and mortality at day 28.

\section{Statistical methods}

All the analyses will be performed by the study statistician, in accordance with the International Conference on Harmonisation and Good Clinical Practice guidelines. A predefined statistical analysis plan will be followed. The analysis will be performed on an intention-to-treat basis after validation by a blind review committee of the inclusion and exclusion criteria for each patient. All the analyses will be conducted by the biostatistics department of the Poitiers University Hospital using statistical software (SAS, V.9.3; SAS Institute; Cary; North Carolina, USA, and R V.2.14.1). A two-tailed $\mathrm{P}$ value of less than 0.05 will be considered as indicating statistical significance.

\section{Descriptive analysis of patient groups at baseline}

The continuous variables will be summarised with the classic parameters of descriptive analysis (median, interquartile ranges and extreme values or mean and $\mathrm{SD})$, while indicating the number of missing data. The category variables will be presented in the form of absolute frequency and percentage in each modality.

The criteria of subject selection will be verified on the basis of the data recorded in the case reports. Wrongly included subjects such as those lost to follow-up will be described. Deviations from the protocol will be described and analysed on a case-by-case basis.

\section{Analysis pertaining to the main criteria of evaluation}

The percentages of patients having presented an episode of severe hypoxaemia during the intubation procedure will be compared between the two groups by means of the $\mathrm{X}^{2}$ test.

The different parameters that would be potentially predictive of failed preoxygenation will be considered by means of the Student's t-test (or the Mann-Whitney $\mathrm{U}$ test, if necessary) for continuous quantitative variables and by means of the $\mathrm{X}^{2}$ test (or Fisher's exact test) for qualitative variables.

The analysis will subsequently be completed by multivariate logistic regression. The maximal model will include all the variables associated with the dependent variable $(\mathrm{P}<0.20)$ as well as the relevant variables according to the data collected in the literature (forced variables), especially treatment received before randomisation and hypercapnia. The model will be simplified according to a step-by-step procedure of elimination in descending order; only the variables associated with the dependent variable (threshold limit value: $5 \%$ ) and the forced variables will be conserved.

\section{Analysis pertaining to the secondary criteria of evaluation}

Secondary criteria of evaluation will be compared between the two treatment groups by means of the Student's t-test (or the Mann-Whitney U test, if necessary) for continuous quantitative variables and by means of the $\mathrm{X}^{2}$ test (or Fisher's exact test) for qualitative variables.

Subgroup analysis, which include patients with $\mathrm{PaO}_{2} /$ $\mathrm{FiO}_{2}$, will be conducted to analyse outcomes in patients with severe hypoxaemia.

\section{METHODS: MONITORING \\ Data monitoring}

Before starting patient enrolment, all physicians and other healthcare workers in the ICU attend formal training sessions on the study protocol and data collection. An investigator at each centre is responsible for daily patient screening, enrolling patients in the study, ensuring adherence to the protocol and completing the e-CRF. Research assistants regularly monitor all the centres on site to check adherence to the protocol and the accuracy of the data recorded.

\section{Harms}

The investigator is allowed to temporarily or permanently discontinue the participation of a patient in the study for any reason that would optimally serve the interests of 
the subject, particularly in case of serious adverse events suspected to be associated with the type of preoxygenation used.

\section{Auditing}

An independent Data and Safety Monitoring Board, composed of four experts (Sophie Duranton, Cendrine Godet, Xavier Drouot and Florence Boissier), will monitor the safety of the trial.

\section{ETHICS AND DISSEMINATION Consent or assent}

The patient is included after having provided a written informed consent to the investigator according to the decision of the central ethics committee. If the patient is not able to understand the information given, he/she can be included if the same procedure is completed with a next of kin. After the patient's recovery, he/she will be asked if he/she agrees to continue the trial.

\section{Confidentiality}

Data will be handled according to French law. All original records will be archived at trial sites for 15 years. The clean database file will be anonymised and kept for 15 years.

\section{Declaration of interest}

The FLORALI-2 study is an investigator-initiated trial funded by the French Ministry of Health obtained in 2014 from a national hospital clinical research programme (Programme Hospitalier de Recherche Clinique National 2014). A scientific committee including J-PF, J-DR and AWT conceived, drafted and wrote the project. The European research network REVA has endorsed the study project. The study is promoted by the University Hospital of Poitiers. The firm Fisher \& Paykel Healthcare provides the high-flow oxygen therapy equipment to all the participating centres but has no other involvement in the study.

\section{Access to data}

All investigators will have access to the final data set. Participant-level data sets will be made accessible on a controlled access basis.

\section{Dissemination policy}

Findings will be published in peer-reviewed journals and presented at local, national and international meetings and conferences to publicise and explain the research to clinicians, commissioners and service users.

\section{DISCUSSION}

Severe oxygen desaturation, commonly defined as a drop in $\mathrm{SpO}_{2}$ below $80 \%$, is a life-threatening complication frequently encountered during intubation of critically ill patients in the ICU. ${ }^{18}$ Preoxygenation is of great importance to prevent oxygen desaturation, especially in patients with hypoxaemia. ${ }^{5}$ Although breathing through a valve-bag facemask is the usual procedure for preoxygenation in the operating room, other strategies have been proposed in ICUs, especially NIV in patients with acute hypoxaemic respiratory failure. However, to date, only one RCT has compared NIV for preoxygenation with standard oxygen using valve-bag facemask in ICU patients needing intubation. ${ }^{14}$ In this study, which included 53 patients with hypoxaemia from two units, Baillard and colleagues reported that NIV improved oxygenation at the end of preoxygenation and markedly decreased the proportion of patients with severe oxygen desaturation during intubation (from $46 \%$ to only $7 \%, \mathrm{P}<0.01$ ) as compared with standard oxygen. ${ }^{14}$ NIV may prevent the severe hypoxaemia probably due to higher $\mathrm{FiO}_{2}$ and to increased end-expiratory lung volume induced by PEEP. However, episodes of profound desaturation were not fully suppressed in the NIV group, and some patients experienced $\mathrm{SpO}_{2}$ as low as $70 \%$, suggesting the detrimental role of removing the facemask to allow for the laryngoscopy.

Several studies have compared HFNC with standard oxygen for preoxygenation of patients with hypoxaemia. ${ }^{9-12}$ HFNC may provide expiratory positive airway pressure and maintain oxygenation at high $\mathrm{FiO}_{2}$ during the apneic phase of intubation. ${ }^{20}{ }^{21}$ In a prospective before-after study including 101 patients, the use of HFNC with a flow of $60 \mathrm{~L} / \mathrm{min}$ resulted in higher $\mathrm{SpO}_{2}$ at the end of preoxygenation and lower incidence of severe desaturation below $80 \%$ during intubation than with standard oxygen through a non-rebreathing mask: only $2 \%$ with HFNC versus $14 \% \quad(\mathrm{P}=0.03) .{ }^{9}$ However, these promising results have not been confirmed in the different RCT carried out to date. ${ }^{10-12}$ In a recent RCT including 150 non-selected patients requiring intubation, oxygenation during the apneic phase did not prevent severe desaturation $<80 \%$, with a rate of $16 \%$ versus $25 \%$ in the group with standard preoxygenation without apneic oxygenation $(\mathrm{P}=0.22) .{ }^{10}$ However, oxygenation during the apneic phase was performed using a flow of only $15 \mathrm{~L} / \mathrm{min}^{10}{ }^{10}$ which is quite far from that which can be provided with HFNC. In another RCT including 119 patients with hypoxaemia, HFNC set with a flow of $60 \mathrm{~L} /$ min did not prevent desaturation and the lowest $\mathrm{SpO}_{2}$ during intubation did not differ between the two strategies. ${ }^{12}$ In a recent RCT including 40 ICU patients, a significant drop of $\mathrm{SpO}_{2}$ was observed with standard oxygen during apnea after the induction of anaesthesia, whereas this was not the case with HFNC. ${ }^{11}$ Of note, none of these studies mentioned the practice of jaw thrust, which is crucial to ensure patients' upper airways, a critical determinant of efficient apneic oxygenation. ${ }^{7}$ This may explain, at least in part, some of the discrepancies among studies which failed to show a benefit of HFNC and those that did. The use of HFNC to manage acute hypoxaemic respiratory failure in ICU has been growing. In a patient already treated with HFNC for acute respiratory failure, it seems reasonable that preoxygenation should not be performed with standard oxygen, and would be better 
carried out with HFNC or NIV. Because HFNC has clearly been shown to be superior to standard oxygen to manage patients with acute hypoxaemic respiratory failure, ${ }^{21-23}$ we believed that it would not be reasonable to perform preoxygenation with standard oxygen in a patient already treated with HFNC or NIV. Therefore, to avoid potential deleterious effects of standard oxygen, and to allow the use of ventilatory support, we decided to use HFNC in the control group.

A recent study showed that NIV associated with HFNC seemed to be more beneficial compared with NIV alone during the preoxygenation of patients with hypoxaemia. ${ }^{16}$ In another study, no difference was found between preoxygenation with NIV and HFNC. ${ }^{15}$ However, the small sample size of these two studies does not allow for conclusions, and in order to clearly identify the best strategy for preoxygenation in ICU, we planned to conduct this large RCT using NIV alone in the interventional group. The FLORALI-2 study was empowered to investigate the effectiveness of NIV in decreasing occurrence of severe oxygen desaturation episodes, defined as $\mathrm{SpO}_{2}<80 \%$ as in the majority of the studies evaluating the best method for preoxygenation. As NIV is clearly not the standard care for preoxygenation in clinical practice, we did not design this study as a non-inferiority study but rather as a superiority study.

One limitation of the study is that investigators are aware of the inclusion group. However, given the characteristics of the two strategies under evaluation, a double-blind trial is not possible. Second, the assessment of severe hypoxaemia during the intubation procedure should be more accurate by analysing blood gas sample rather than $\mathrm{SpO}_{2}$. However, this could delay the tracheal intubation in an emergent context and reduce the feasibility and pertinence of the study by including less severe patients. In order to improve the measurement reliability in all centres, a dedicated portable $\mathrm{SpO}_{2}$ monitor with single-patient-use digital sensors is used for each eligible patient to ensure that all centres have equivalent monitoring, and secondarily, the downloaded data will be reviewed so as to analyse the events occurring during intubation procedure. This represents a strength of this study, which will be the largest study comparing two preoxygenation strategies in this field. To minimise bias, adjustments will be made after multivariate logistic regression by including variables independently associated with severe oxygen desaturation, and especially according to the difficulty of intubation.

In conclusion, the FLORALI-2 trial is an investigator-initiated pragmatic RCT empowered to test the hypothesis that NIV in comparison to HFNC alone helps to decrease episodes of severe hypoxaemia during the intubation procedure of ICU patients with hypoxaemic respiratory failure requiring mechanical ventilation. The FLORALI-2 trial will also assess the impact of NIV and HFNC for preoxygenation on intubation-related complications and ICU outcomes.

\section{Author affiliations}

${ }^{1}$ Réanimation Médicale, CHU de Poitiers, Poitiers, France

${ }^{2}$ Faculté de Médecine et de Pharmacie de Poitiers, Université de Poitiers, Poitiers, France

${ }^{3}$ INSERM, CIC-1402, équipe 5 ALIVE, Poitiers, France

${ }^{4}$ Service de Réanimation Médico-Chirurgicale, AP-HP, Hôpital Louis Mourier, Colombes, France

${ }^{5}$ Université Paris Diderot, UMR IAME 1137, Sorbonne Paris Cité, Paris, France

${ }^{6}$ INSERM, IAME 1137, Paris, France

${ }^{7}$ Department of Biostatistics, INSERM, CIC-1402, Poitiers, France

${ }^{8} \mathrm{CIC}-1402$, Poitiers, France

Collaborators Members of boards and committees of the study. Members of the scientific committee: Jean-Pierre Frat, Jean-Damien Ricard and Arnaud W Thille conceived, drafted and wrote the project. Members of the data monitoring committee: Céline Delétage-Métreau and Morgane Olivry. Members of the blindreview board: Stéphanie Ragot, Severine Clerjaud, Jean-Pierre Frat, Marie Bonnin, Céline Delétage-Métreau and Morgane Olivry. Members of the safety monitoring board: Sophie Duranton, Florence Boissier, Cendrine Godet and Xavier Drouot.

Contributors J-PF, J-DR, RC, RR and AWT designed together the study, and wrote together the manuscript. SR provided substantial contributions to the conception and design of the study, and wrote with J-PF the statistical analysis plan and estimated the sample size. J-PF, J-DR, RC, RR, SR and AWT contributed for drafting the work, revising it critically for important intellectual content and approved the final version of the manuscript. All authors give their agreement to be accountable for all aspects of the work, and ensure the accuracy and integrity of any part of the work.

Funding The study was funded by the 'Programme Hospitalier de Recherche Clinique National 2014' of the French Ministry of Health. The study promoter is the University Hospital of Poitiers, Poitiers, France.

Disclaimer The firm Fisher \& Paykel provides to all the participating centres the high-flow oxygen therapy equipment but has no other involvement in the study.

Competing interests J-PF reports consulting fees from Fisher and Paykel. J-DR reports travel expenses coverage to attend scientific meetings by Fisher \& Paykel.

Ethics approval The study has been approved by the central ethics committee (Ethics Committee Ouest III, Poitiers, France) with the registration number 2015A00530-49 Soins courants (24 June 2015).

Provenance and peer review Not commissioned; externally peer reviewed

Open Access This is an Open Access article distributed in accordance with the Creative Commons Attribution Non Commercial (CC BY-NC 4.0) license, which permits others to distribute, remix, adapt, build upon this work non-commercially, and license their derivative works on different terms, provided the original work is properly cited and the use is non-commercial. See: http://creativecommons.org/ licenses/by-nc/4.0/

(C) Article author(s) (or their employer(s) unless otherwise stated in the text of the article) 2017. All rights reserved. No commercial use is permitted unless otherwise expressly granted.

\section{REFERENCES}

1. Jaber S, Amraoui J, Lefrant JY, et al. Clinical practice and risk factors for immediate complications of endotracheal intubation in the intensive care unit: a prospective, multiple-center study. Crit Care Med 2006;34:2355-61.

2. Griesdale DE, Bosma TL, Kurth T, et al. Complications of endotracheal intubation in the critically ill. Intensive Care Med 2008;34:1835-42.

3. Watson PL, Pandharipande P, Gehlbach BK, et al. Atypical sleep in ventilated patients: empirical electroencephalography findings and the path toward revised ICU sleep scoring criteria. Crit Care Med 2013;41:1958-67.

4. Dessap AM, Roche-Campo F, Launay JM, et al. Delirium and circadian rhythm of melatonin during weaning from mechanical ventilation: an ancillary study of a weaning trial. Chest 2015;148:1231-41.

5. Peñuelas $\mathrm{O}$, Frutos-Vivar F, Fernández $\mathrm{C}$, et al. Characteristics and outcomes of ventilated patients according to time to liberation from mechanical ventilation. Am J Respir Crit Care Med 2011;184:430-7. 
6. Nimmagadda U, Salem MR, Crystal GJ. Preoxygenation: physiologic basis, benefits, and potential risks. Anesth Analg 2017;124:507-17.

7. Ricard JD. Hazards of intubation in the ICU: role of nasal high flow oxygen therapy for preoxygenation and apneic oxygenation to prevent desaturation. Minerva Anestesiol 2016;82:1098-106.

8. Sim MA, Dean P, Kinsella J, et al. Performance of oxygen delivery devices when the breathing pattern of respiratory failure is simulated. Anaesthesia 2008;63:938-40.

9. Miguel-Montanes R, Hajage D, Messika J, et al. Use of high-flow nasal cannula oxygen therapy to prevent desaturation during tracheal intubation of intensive care patients with mild-to-moderate hypoxemia. Crit Care Med 2015;43:574-83.

10. Semler MW, Janz DR, Lentz RJ, et al. Randomized trial of apneic oxygenation during endotracheal intubation of the critically III. Am J Respir Crit Care Med 2016;193:273-80.

11. Simon M, Wachs C, Braune S, et al. High-flow nasal cannula versus bag-valve-mask for preoxygenation before intubation in subjects with hypoxemic respiratory failure. Respir Care 2016;61:1160-7.

12. Vourc'h M, Asfar P, Volteau C, et al. High-flow nasal cannula oxygen during endotracheal intubation in hypoxemic patients: a randomized controlled clinical trial. Intensive Care Med 2015;41:1538-48.

13. L'Her E, Deye N, Lellouche F, et al. Physiologic effects of noninvasive ventilation during acute lung injury. Am J Respir Crit Care Med 2005;172:1112-8.

14. Baillard C, Fosse JP, Sebbane M, et al. Noninvasive ventilation improves preoxygenation before intubation of hypoxic patients. Am J Respir Crit Care Med 2006;174:171-7.

15. Besnier E, Guernon K, Bubenheim M, et al. Pre-oxygenation with high-flow nasal cannula oxygen therapy and non-invasive ventilation for intubation in the intensive care unit. Intensive Care Med 2016:42:1291-2.

16. Jaber S, Monnin M, Girard M, et al. Apnoeic oxygenation via highflow nasal cannula oxygen combined with non-invasive ventilation preoxygenation for intubation in hypoxaemic patients in the intensive care unit: the single-centre, blinded, randomised controlled OPTINIV trial. Intensive Care Med 2016;42:1877-87.

17. Coudroy R, Thille AW, Drouot X, et al. How to assess FiO2 delivered under oxygen mask in clinical practice? Annals of Intensive Care 2017;7:196.

18. De Jong A, Molinari N, Terzi N, et al. Early identification of patients at risk for difficult intubation in the intensive care unit: development and validation of the MACOCHA score in a multicenter cohort study. Am J Respir Crit Care Med 2013;187:832-9.

19. Cormack RS, Lehane J. Difficult tracheal intubation in obstetrics. Anaesthesia 1984;39:1105-11.

20. Adnet F, Borron SW, Racine SX, et al. The intubation difficulty scale (IDS): proposal and evaluation of a new score characterizing the complexity of endotracheal intubation. Anesthesiology 1997;87:1290-7.

21. Frat JP, Thille AW, Mercat A, et al. High-flow oxygen through nasal cannula in acute hypoxemic respiratory failure. N Engl $J$ Med 2015;372:2185-96.

22. Frat JP, Ragot S, Girault C, et al. Effect of non-invasive oxygenation strategies in immunocompromised patients with severe acute respiratory failure: a post-hoc analysis of a randomised trial. Lancet Respir Med 2016;4:646-52.

23. Sztrymf B, Messika J, Bertrand F, et al. Beneficial effects of humidified high flow nasal oxygen in critical care patients: a prospective pilot study. Intensive Care Med 2011;37:1780-6. 